\title{
Effect of Gas Composition on the Ablation Behavior
}

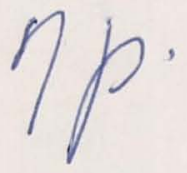
of a Charring Material

\author{
Nick S. Vojvodich* and Ronald B. Pope* \\ NASA Ames Research Center, Moffett Field, Calif.
}

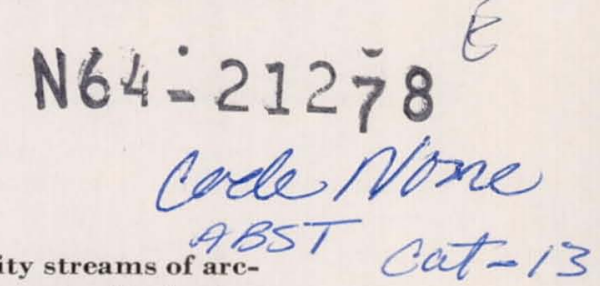

The ablation behavior of a charring material was evaluated in high-velocity streams of archeated air, nitrogen, and argon. The purpose of the investigation was to determine 1) whether chemical reactions occur between the material and its environment, 2) whether the reactions are homogeneous and/or heterogeneous, and 3) what the influence of the reactions is upon the heating rate to the surface of the material. The behavior of the material proved to be highly sensitive to the composition of the test gas. For air, both heterogeneous and homogeneous reactions were found to occur, and the surface heating rates resulting from each type of reaction were evaluated and compared to theory. In the case of homogeneous reactions, experiment was higher than theory, whereas the opposite was true for the heterogeneous reactions.

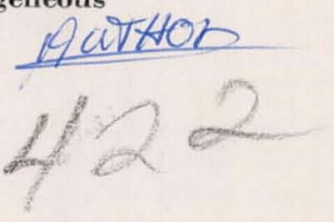

\section{Nomenclature}

$D^{*}=$ throat diameter

$E \quad=$ constant as defined in Eq. (A9)

$H \quad=$ enthalpy

$h_{A}=$ intrinsic heat capacity of pyrolysis

$h_{C}=$ heat of combustion

$K=$ mass fraction of oxygen

$k=$ thermal conductivity

$L \quad=$ initial length of test sample

$M \quad=$ average molecular weight

$m \quad=$ mass

$\dot{m}=$ mass-loss rate

$P \quad=$ pressure

$\dot{q}_{\mathrm{comb}}=$ heating rate resulting from chemical reactions

$\dot{q}_{\text {conv }}=$ hot-wall convective heating rate with transpiration, $\psi \dot{q}_{h w}$

$\dot{q}_{h w}=$ hot-wall convective heating rate without transpiration

$\dot{q}_{i}=$ net heating rate to gas-surface interface, $\dot{q}_{\text {conv }}+\dot{q}_{\text {comb }}+$ $\dot{q}_{r}$

$\dot{q}_{0}=$ cold-wall convective heating rate without transpiration

$\dot{q}_{r} \quad=$ radiative heating from boundary-layer gases to surface

$\dot{q}_{\mathrm{rr}}=$ radiative heating from surface, $\epsilon \sigma T_{w}{ }^{4}$

$R_{\text {eft }}=$ effective nose radius

$T=$ surface temperature

$t \quad=$ time

$u \quad=$ gas velocity parallel to surface

$v \quad=$ gas velocity normal to surface

$x=$ direction parallel to surface

$y \quad=$ direction normal to surface

$=$ surface recession rate

$=$ exponent defined in Eq. (A9)

$=$ transpiration factor

$=$ change

$=$ char thickness

$=$ emissivity

$=$ density

$=$ Stefan-Boltzmann constant

$=\dot{q}_{\text {oonv }} / \dot{q}_{h w}$

Subscripts

$a \quad=$ air

$=$ char

$=$ nitrogen

$=$ pyrolysis

$=$ total

= vapor

Presented as Preprint 63-465 at the AIAA Conference on Physics of Entry into Planetary Atmospheres, Cambridge, Mass., August 26-28, 1963; revision received January 15, 1964.

* Research Scientist. Member AIAA.

$$
\begin{aligned}
w & =\text { wall } \\
\infty & =\text { freestream }
\end{aligned}
$$

\section{Introduction}

A SIGNIFICANT problem in heat protection research arises from the chemical interaction between an ablation system and the boundary-layer flow. The stagnation region of a typical thermal protection system consisting of a porous char layer, through which ablation gases are transpired, is represented in Fig. 1. It is noted that the heating environment may be influenced by exothermic reactions occurring at the high-temperature surface of the char and in the boundary layer. The extent of these reactions is strongly influenced by the diffusion rate of the chemically active boundary-layer species to the reaction zones.

The effect of chemical reactions upon the behavior of a pure material, such as graphite, has been studied extensively. ${ }^{1-5}$ Only limited theories ${ }^{6}$ and experiments ${ }^{7.8}$ have considered the chemical reactions between a charring material and the boundary-layer species. From the available data, it is not possible to deduce the type of reaction (homogeneous and/or heterogeneous) or the amount of heat transferred to the surface by the reaction. Accordingly, an experimental program was undertaken to study the response of a charring material in high-enthalpy streams of air, nitrogen, and argon over a wide range of convective heating rates characteristic of conditions encountered upon planetary entry. This paper presents the preliminary results obtained to date and discusses these results in terms of the physics and chemistry of the interaction between the charring material, its vapors, and the boundary-layer gases.

\section{Experimental Equipment}

\section{Facility}

The tests were performed in the facility shown schematically in Fig. 2. Test gases of either air, nitrogen, or argon were heated to high energy levels by a fully water-cooled electric are heater, ${ }^{9-12}$ which operates with thoriated tungsten electrodes and yields a gas with a low contamination level. The heated gas was expanded from a plenum chamber through a water-cooled, supersonic nozzle. Five different nozzles were used, four of which were conical with exit diameters of nominally $1,2,3$, and 6 in., the fifth being a contoured nozzle with a 4 -in. exit. The throat diameter 
was 0.468 in. for all the nozzles. The supersonic stream was discharged as a free jet into a vacuum chamber that was evacuated by a steam-ejector vacuum system.

\section{Test Models and Apparatus}

A representative high-temperature charring composite material was selected for the investigation. The particular material was a low-density $\left(36 \mathrm{lb} / \mathrm{ft}^{3}\right)$ phenolic-nylon material composed, by weight, of $50 \%$ nylon, $25 \%$ phenolic resin, and $25 \%$ phenolic microballoons.

Two model configurations, a flat-faced cylinder and a hemisphere-cylinder with afterbody test segments, were used to obtain a wide range of convective heating rates. Sketches of these models are shown in Fig. 3. The flat-faced models had either a $\frac{1}{2}$ or $\frac{3}{4}$-in. diam and were 0.4 in. long. These models were supported by thin-walled stainless-steel tubing. The diameter of the hemisphere-cylinder models was $\frac{5}{8}$ in.; the construction details and method of support are described in Ref. 9.

Cold-wall heating rates were measured with heat-sink, transient-type, copper calorimeters having external dimensions identical to the ablation models. Details of the calorimeter design have been presented in Ref. 9. The wall pressure at the stagnation region was measured with a water-cooled, impact pressure tube, and a strain-gage-type pressure cell with a pressure range of 0 to 3 psia. The surface temperature of each ablating specimen was monitored during the entire heating period with a commercial, total-radiation pyrometer.

\section{Test Conditions and Procedure}

The energy level of the supersonic stream was changed by varying the total input energy from approximately 40 to 80 $\mathrm{kw}$ at a constant reservoir pressure of $\frac{1}{3} \mathrm{~atm}$. The freestream enthalpy was determined first from measurements of mass flow, electrical input energy, and heat losses to the are heater and nozzle, and second from the measurements of impact pressure and heating rates used in conjunction with the correlation developed by Fay and Riddell, ${ }^{13}$ which predicts the convective heating rate to a blunt body for the case of a laminar boundary layer in chemical equilibrium. Although at the pressure levels of these tests the test stream was not in chemical equilibrium, ${ }^{10}, 11$ the application of the second technique for determining enthalpy has been shown to be reliable ${ }^{12}$ when the surface of the calorimeter is a highly catalytic material such as copper. ${ }^{11}$ The enthalpies determined by these two techniques were in good agreement and varied from 4200 to $8500 \mathrm{Btu} / \mathrm{lb}$ for air and nitrogen. The enthalpy determined for argon was $3000 \mathrm{Btu} / \mathrm{lb}$.

The test conditions, cold-wall heating rate, model pressure, and stream enthalpy are summarized in Fig. 3. As indicated, the cold-wall heating rates varying from 25 to $200 \mathrm{Btu} /$ $\mathrm{ft}^{2}$-sec were obtained with the flat-faced models in the conical
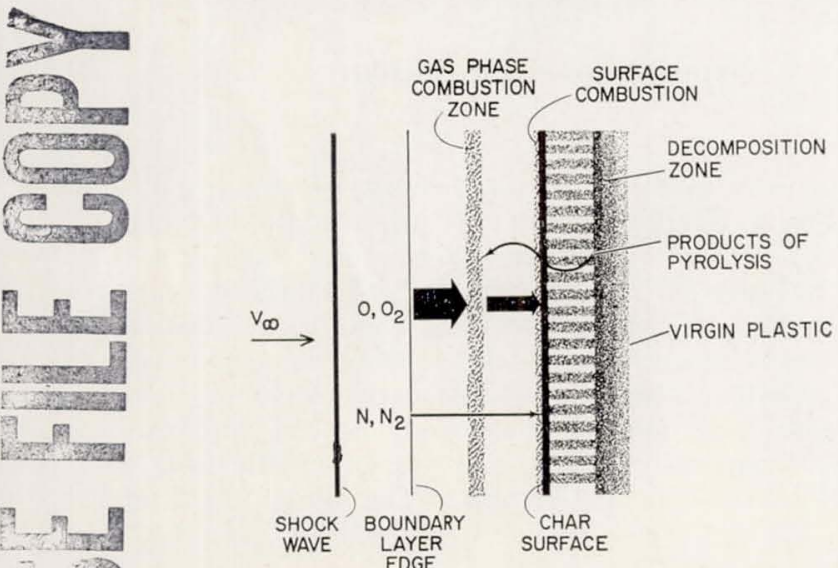

Fig. 1 Physical representation of problem.

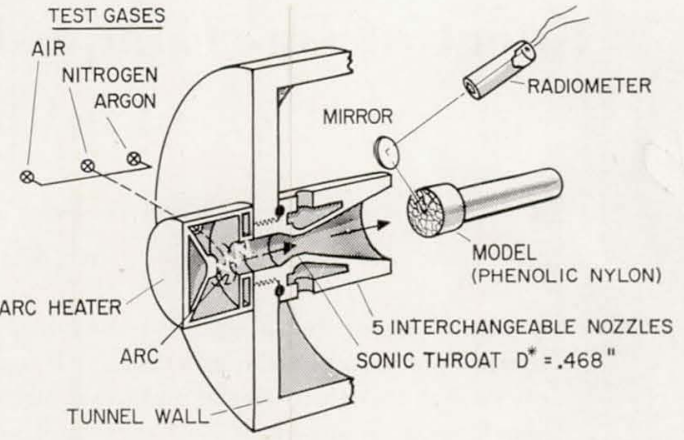

NOTE:UNIT WATER COOLED

Fig. 2 Test facility.

nozzles. This wide range of convective heating rates at comparable stream enthalpies was made possible by the use of the various nozzles previously described. The heating rates varying from 3 to $13 \mathrm{Btu} / \mathrm{ft}^{2}$ sec were obtained with the afterbody models in the 4 -in. contoured nozzle. Thus, heating rates of approximately two orders of magnitude were covered by this investigation. Since the afterbody pressures were too low to be measured with the available instrumentation, they were calculated by the method of Ref. 14 .

To provide satisfactory stream conditions over the length of the test models, it was necessary to maintain a balance between the nozzle wall pressure at the exit and the test chamber pressure. This balance was provided by throttling the steam-ejector system.

For the purpose of measuring the surface temperature, an image of the test region of the specimen was focused onto the pyrometer by means of a first surface mirror located at the side of the nozzle (see Fig. 2). The mirror and a commercial blackbody temperature source were used to calibrate the pyrometer. The temperatures reported herein were not corrected for emissivity effects, but the emissivity for this type of charring material has been reported to range from approximately 0.85 to $0.80 .6,15,16$ Therefore, the true temperature is only from 4 to $5 \frac{1}{2} \%$ higher than the blackbody temperature reported.

The procedure for testing in each nozzle was first to measure cold-wall heating rates and pressures over the range of stream enthalpies, then to expose a number of ablation models to identical stream conditions for various times, and finally to repeat the cold-wall measurements. After each ablation model was weighed and its initial length determined, it was located at the center line of the stream behind a protective shield. When the test chamber had been evacuated, the arc was initiated, the stream was stabilized, and the shield was quickly removed. The are was extinguished at a predetermined time, following which the model was removed and weighed and its length measured.

\section{Results and Discussion}

\section{Experimental Results}

Typical variations of the surface temperature $T_{w}$ and change in mass $\Delta m$ are compared for air (half-filled symbols) and nitrogen (open symbols) streams in Figs. $4 \mathrm{a}$ and $4 \mathrm{~b}$. Different symbols indicate tests of varying duration. It is noted that, at both heating rates, the gas composition had a sizable effect upon both the surface temperature and the change in mass. Furthermore, the good agreement between the temperature histories measured in air for various exposure times indicates that stream conditions were repeatable.

The temperature data for all of the test conditions are shown in Fig. 5 as a function of total stream enthalpy. (The value plotted is the arithmetic mean of the temperatures for the longest and shortest test periods used in determining 


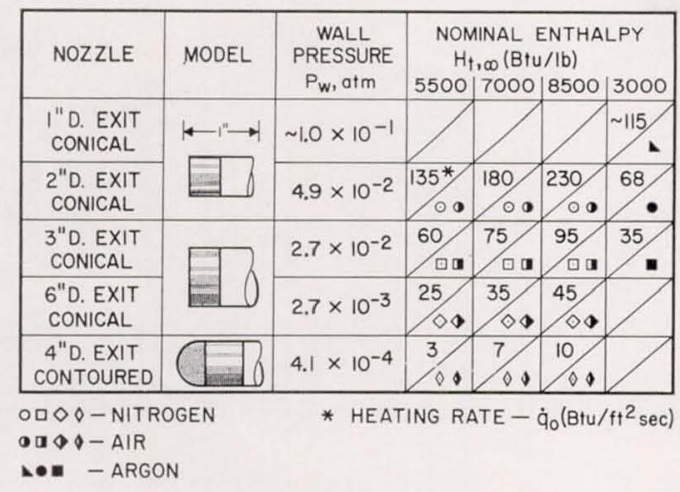

Fig. 3 Test conditions and models.

the mass-loss rate.) Sets of data are plotted for four different model wall pressures. For each pressure level, the effect of gas composition is clearly demonstrated. These differences cannot be explained by differences in cold-wall heating rates, since, as shown in Fig. 3, the cold-wall heating rates at comparable enthalpy and pressure were identical. These results, therefore, show that exothermic chemical reactions occurred between the air and the injected gases and/or the char surface.

Having established the importance of gas composition on the surface temperature of the material, let us now consider the mass-loss measurements. These consisted of the total mass-loss rate and its two components: char removal $\dagger$ and the vapors produced by pyrolysis. These results are presented in Fig. 6. It will be noted that the total mass-loss rate and its two components were always greater in air than in nitrogen. This was true at all pressure levels. Once again the effect of gas composition upon the measurements is attributable to chemical reactions rather than to heatingrate differences.

There are two points of interest with respect to char mass-loss rates. One is that these rates were about an order of magnitude less than the corresponding total rate, and the other is that the rates in nitrogen were appreciable.

The two types of measurements - surface temperature and mass-loss rate- have demonstrated significant effects of gas composition on the response of the material. These effects have been attributed to chemical reactions. The nature of these reactions and the resulting surface heating are considered in the following section.

\section{Determination of Reaction Zones and Heating}

First, we will examine in detail the data on char mass-loss rate to determine whether or not chemical reactions influence the char removal. These data, which have been normalized by the square root of the wall pressure divided by the effective nose radius, ${ }^{17}$ have been plotted in Fig. 7 as a function of wall temperature. $\ddagger$ This form of presentation is appropriate when the reaction of the surface with the environment is controlled primarily by diffusion of oxygen to the surface. Available information ${ }^{2}$ indicates that, at temperatures below $2000^{\circ} \mathrm{R}$, the surface recession of graphite is limited by the reaction rate kinetics, whereas between $2000^{\circ}$ and $5000^{\circ} \mathrm{R}$ the process is diffusion rate-limited. At temperatures in excess of $5000^{\circ} \mathrm{R}$, Scala and Gilbert ${ }^{18}$ have recently shown that the surface recession of graphite is a strong function of temperature as well as pressure because of the importance

$\dagger$ The char mass-loss rate was determined from measurements of the recession rate $\dot{y}$ and the char density, which was found to be $12 \pm 1 \mathrm{lb} / \mathrm{ft}^{3}$ for all test conditions.

$\ddagger$ As indicated in Fig. 3, the pressure was varied over a wide range; however, only two model radii were employed, and, hence, additional tests with larger models are required in order to establish the dependence of $\dot{m}_{c}$ on $R_{\text {eff }}$.

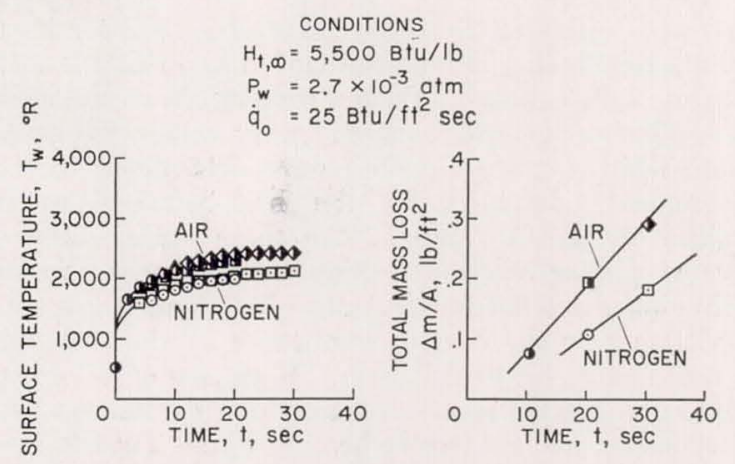

Fig. 4a Typical measurements: low heating rate.

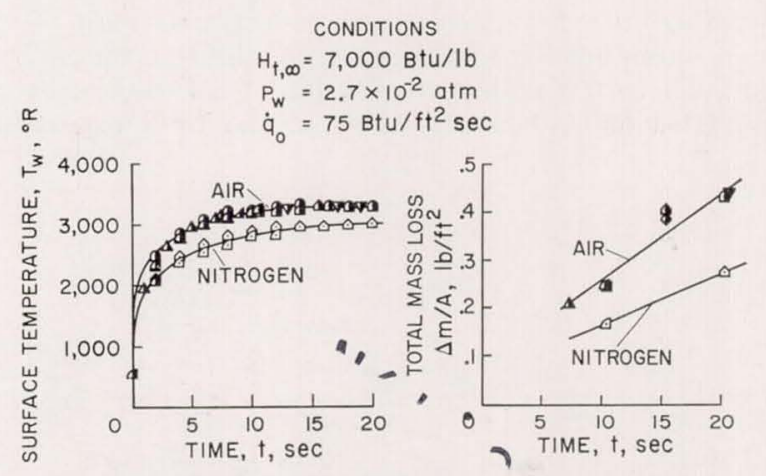

Fig. 4b Typical measurements: mode̊rafe heating rate.

of $\mathrm{C}-\mathrm{N}$ reactions at elevated temperatures. To 2determine, in fact, if there were chemical reactions occurring between the nitrogen and char for the particular conditions of these tests, a number of models were exposed to the chemically inert stream of argon. The resulting data points are shown by the filled symbols on the figure. Good agreement of these points for argon with the data points for nitrogen show that the nitrogen was chemically inert. This result is in good agreement with the work of Zinman, ${ }^{19}$ who reported the rates of reaction between carbon and nitrogen to be very slow at temperatures below $5000^{\circ} \mathrm{R}$. Mass-loss rate of char for these two gases is, perhaps, attributable to a combination of mechanical and thermal stresses rather than to chemical erosion. An alternate mechanism for this removal of char in the inert atmospheres, chemical reactions between the pyrolyzed gases and the char, has been suggested by John Parker of Ames. In contrast to the results obtained in the chemically inert streams, it is noted that the results in air are higher by as much as a factor of 3 , indicating that ehemical reaction occurred at the surface in the air environment.

With the amount of char removal by combustion established, and assuming a reasonable value of the heat of combustion, one is in a position to estimate the heat input due to surface combustion. A comparison of these calculated

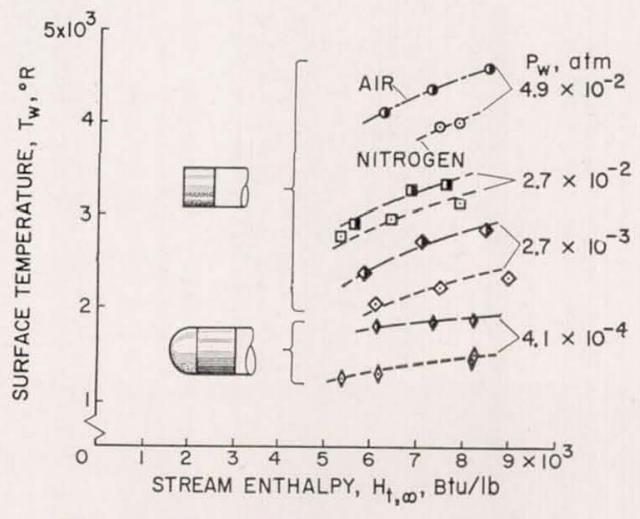

Fig. 5 Temperature measurements. 
values with values obtained for the total combustion heating will indicate whether reactions occurred solely at the surface or also in the gas phase. Such a comparison is provided by Fig. 8. The upper three curves identify values of total combustion heating. These values were determined from the measurements obtained by the heat-balance procedure described in the Appendix. The three lower curves are values of surface reaction heating. They are the result of multiplying the difference in rates of char mass loss for air and nitrogen by the heat of combustion of the char, which was assumed to be $4000 \mathrm{Btu} / \mathrm{lb}$. Both sets of values, that is, total and surface reactions, have been normalized by the corresponding net convective-heating rate. Thus it is seen that the ratio of total combustion heating to the net convective heating varies from a value of unity to about 1.5 , depending upon the pressure. In contrast, the ratio of surface reaction heating to net convective heating ranges from about 0.3 to 0.6 . Thus, the surface reaction heating accounts for only about one-third of the total combustion

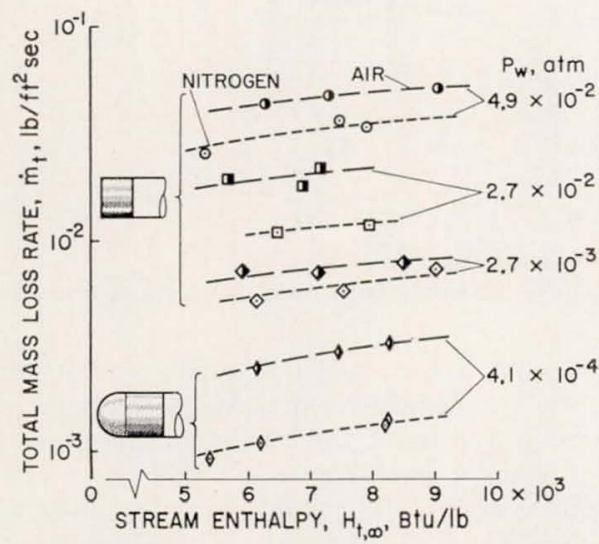

Fig. 6a Mass-loss measurements: total mass-loss rate.

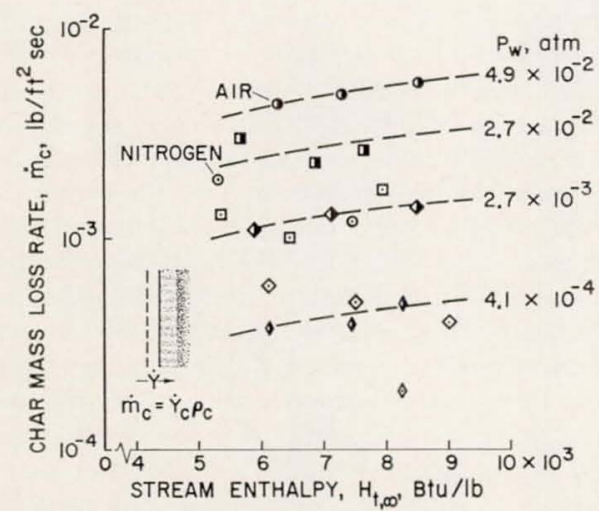

Fig. 6b Mass-loss measurements: char mass-loss rate.

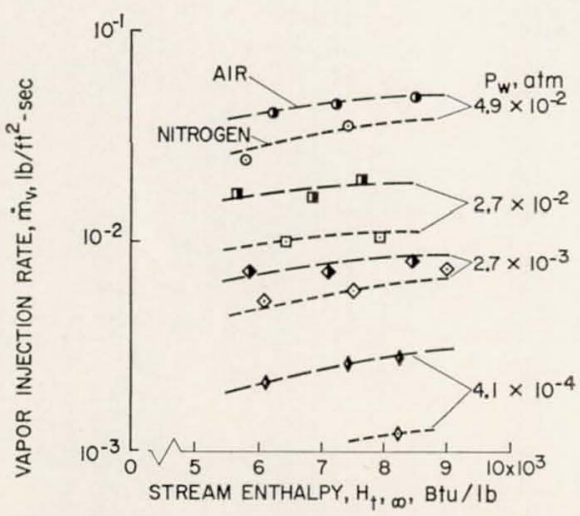

Fig. 6c Mass-loss measurements: vapor injection rate.

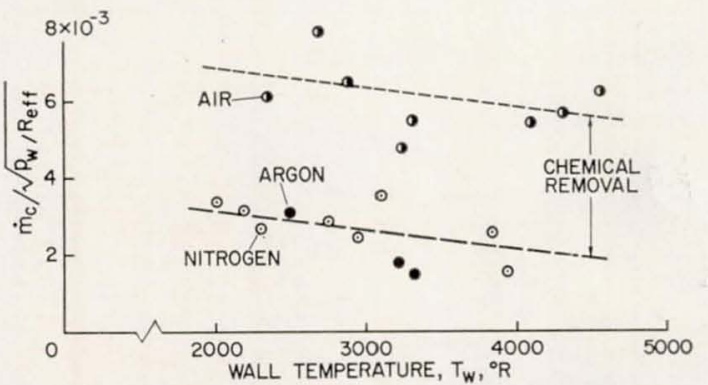

Fig. 7 Effect of gas composition on char removal.

heating. The remainder is attributed to gas-phase-reaction heating.

\section{Comparison of Experiment and Theory}

Having established that both types of reactions occur and that both are appreciable fractions of the net convective heating, it is of interest to determine how well available theory agrees with experiment. Figures 9 and 10 provide comparison of experiment and theory for surface and gasphase-reaction heating, respectively.

In the case of the diffusion-limited surface-reaction heating, the theory used is that of Scala ${ }^{2}$ for the interaction between solid graphite and hypersonic stream, where quantitative answers are obtained from a numerical solution of the nonlinear differential equations. The theoretical values are identified by the solid line in Fig. 9. The experimental values, shown by the dotted line, were obtained from the data shown previously in Fig. 7. It is noted that the theoretical values are one and a half times the experimental values over the full range of wall temperatures.

A possible explanation for the difference between the predicted and measured values is that a basic difference exists between the actual physical process occurring in the experiment and the physical model assumed for the ablation of graphite. Theory assumes that the gases injected into the boundary layer are principally $\mathrm{CO}$ and $\mathrm{CO}_{2}$, which result from chemical reactions between the surface of the graphite and the hypersonic stream. However, for a charring material, light-weight gases are produced by pyrolysis beneath the surface. These gases, some of which are chemically active (e.g., the hydrocarbons $\mathrm{CH}_{4}, \mathrm{C}_{2} \mathrm{H}_{2}$, as well as $\mathrm{H}, \mathrm{H}_{2}$, and $\mathrm{CO}$ ), are subsequently diffused through the char layer and injected into the boundary layer, where, as has been demonstrated, they react with the oxygen diffusing toward the surface. Thus, the unreacted oxygen at the surface which is available for reaction with char is less than that which would be available if the surface were nonporous graphite.

Figure 10 compares theory and experiment for the homogeneous reactions. The curve identified as theory was derived from the work of two groups: Hartnett and Eckert ${ }^{5}$ and Cohen, Bromberg, and Lipkis. ${ }^{4}$ Both groups used the

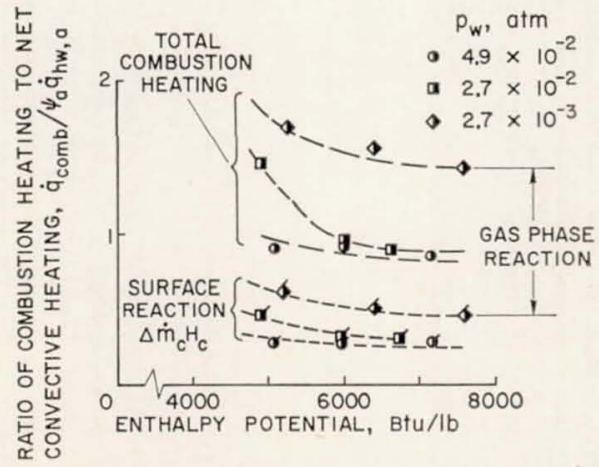

Fig. 8 Variation of combustion heating rate with enthalpy. 


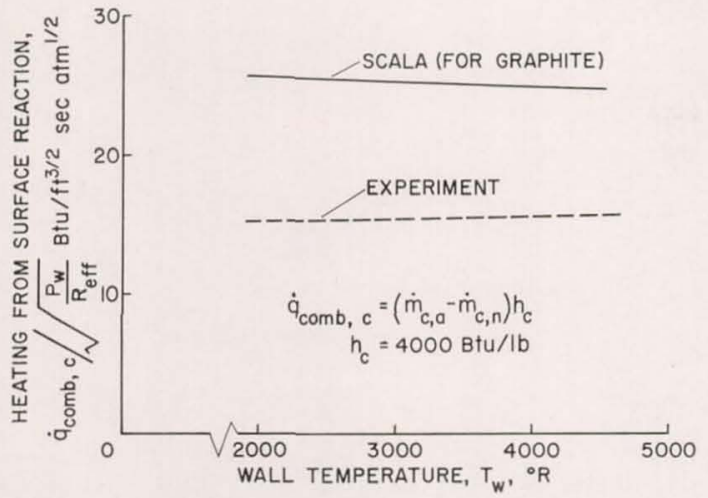

Fig. 9 Comparison of surface reaction heating with theory.

similarity between the diffusion of mass and the transport of energy and obtained the identical result that the effect of combustion is to add a constant term to the boundary-layer enthalpy potential. The increase in the heat flux to the surface due to gas-phase combustion above the value for injection of inert vapors is given by

$$
\dot{q}_{\mathrm{comb}, v} / \psi_{a} \dot{q}_{h w, a}=K h_{c} / \Delta H
$$

where $K$ is the mass fraction of oxygen in the freestream and $h_{C}$ is the heat of combustion for the combustible per pound of oxygen. The reaction of hydrogen with oxygen was used to evaluate this quantity, which is plotted in Fig. 10. Other reactions between the possible carbon-hydrogen vapor mixtures give lower values for the heat of combustion, which, in turn, would give lower estimates of the combustion heating rates. The experimental values shown on Fig. 10 were obtained from data shown previously in Fig. 8 and represent the difference between the total combustion heating and the surface reaction heating. The shaded band in the upper portion of the figure represents the maximum possible combustion heating. In the calculation of these values, all of the oxygen that enters the shock layer was assumed to be consumed in reaction. The range in values arises from the range of test pressures, which, in turn, led to various values of freestream density. It should also be noted that, for this range, the ratio of combustible products to available oxygen always exceeded the stoichiometric ratio.

Having identified the various values of gas-phase combustion heating, it is of interest to compare their relative magnitudes. It is observed that the experimental values are 2 to 5 times higher than the theory; however, the experimental values are considerably below the limiting values of combustion heating.

The possible reasons for these differences are the assumptions used in the theory. In order to make this highly complex problem more tractable, certain simplifying assumptions

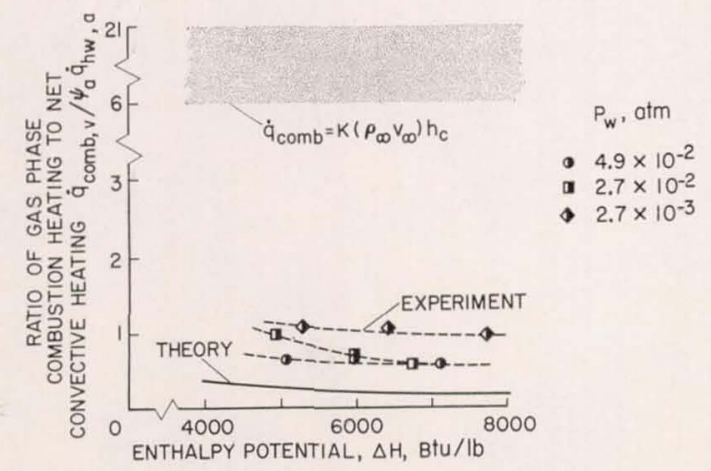

Fig. 10 Comparison of gas-phase combustion heating rate with theory. were made in the theory. These are that the reactions are limited by diffusion, the diffusion coefficients of all species are equal, the frozen Lewis and Prandtl numbers are equal to unity, the reactions occur at a sharp flame front, the location of the flame front is determined by the dimensionless release rate, and the release rate was low. Cohen, Bromberg, and Lipkis ${ }^{4}$ have pointed out that, when the release rate increases beyond a limiting value, the laminar boundarylayer equations become invalid, and the listed assumptions are no longer applicable. Examination of the release rates for both air and nitrogen in this investigation showed them to be large (see Fig. 11). Therefore, it is probable that these large values of release rates account for the disagreement between theory and experiment.

It is of interest, at this point, to cite Barber's measurements of heating rate with transpiration of foreign gases obtained in an arc-heated stream ${ }^{20}$ and compare his results to the data shown in Fig. 10. Barber found the customary reduction of heating rate when inert gases, such as helium, were injected at the stagnation region of the model. However, when chemically active hydrogen was injected into the boundary layer, the heat-transfer rate was greater than that measured for the noninjection case. This additional heating of the surface was attributed to the exothermic, homogeneous reactions occurring between the hydrogen and the boundary-layer species. (The model surface was watercooled copper, which precluded any possibility of heterogeneous surface reactions.) The magnitude of the experimental combustion heating determined by Barber was compared to the theory of Hartnett and Eckert and also found to be higher.

\section{Concluding Remarks}

An investigation of the ablation behavior of a typical charring material conducted in arc-heated streams of air, nitrogen, and argon has indicated that, at a given freestream condition, the surface temperature and total mass-loss rate are highly sensitive to the composition of the test gas. The differences observed between results obtained in nitrogen and air are attributed to exothermic chemical reactions occurring between the air boundary-layer species and the surface of the material and/or the injected vapors.

It is demonstrated that the char mass-loss rate measurements in argon and nitrogen are comparable, which suggests that the nitrogen is chemically inert and the mechanism of char removal for these gases is mechanical or thermal erosion. The corresponding rates obtained in air were higher; hence, it is concluded that heterogeneous combustion occurred.

The total heating due to chemical reactions was evaluated empirically and found to be comparable in magnitude to the net convective heating rate and higher than that which could be accounted for by surface combustion alone. The difference, which represents gas-phase combustion heating, was compared to the theoretical predictions of Hartnett and Eckert and of Cohen, Bromberg, and Lipkis. Both theories underestimated the gaseous combustion heating by a significant amount, possibly because the similarity relationships used in the theories were not valid for the large mass injection rates encountered in the experiment.

The preliminary results of this investigation indicated that the chemical composition of various planetary atmospheres can be expected to influence the response of materials under hypersonic heating conditions. Similar studies performed for gas mixtures that more closely resemble the model atmospheres of Venus and Mars would be desirable for the design of thermal protection systems for entry into these planetary atmospheres. In addition to the gross surface responses of the material reported herein, measurements such as spectrographic surveys of the boundary layer and determination of internal material response are desirable. Such information would provide a more detailed 
description of the basic ablation phenomena, including a definition of the injected vapor species and their molecular weights, the location of the chemical reaction plane in the boundary layer, and an understanding of thermal-degradation mechanism.

\section{Appendix : Evaluation of Total Heating Due to Chemical Reactions}

The total heating rate due to chemical reactions can be determined empirically by performing an energy balance at the ablating surface for both air and nitrogen. For the air tests, the energy balance at the stagnation point for steady-state ablation§ is

$$
\dot{q}_{i}=\dot{q}_{\mathrm{comb}, t}+\dot{q}_{\mathrm{conv}}+\dot{q}_{\tau}=\dot{q}_{r r}+\left[k_{c}(\partial T / \partial Y)\right]_{w}
$$

where

$$
\begin{aligned}
& \dot{q}_{i} \quad=\text { total input heating } \\
& \dot{q}_{\mathrm{comb}, \mathrm{\imath}}=\text { total combustion heating rate consisting }
\end{aligned}
$$

The energy balance at the surface for air can now be written as

$$
\psi \dot{q}_{h w}+\dot{q}_{\mathrm{comb}, t}=\epsilon \sigma T_{w}{ }^{4}+\left[k_{c}(\partial T / \partial Y)\right]_{w}
$$

For the inert stream of nitrogen, $\dot{q}_{\mathrm{comb}, t}=0$, and the energy equation reduces to

$$
\psi \dot{q}_{h w}=\epsilon \sigma T_{w}{ }^{4}+\left[k_{c}(\partial T / \partial Y)\right]_{w}
$$

The difference of Eqs. (A2) and (A3) gives the following equation for $\dot{q}_{\mathrm{comb}, t}$ :

$$
\begin{aligned}
\dot{q}_{\mathrm{comb}, t}=\psi_{n} \dot{q}_{h w, n}- & \psi_{a} \dot{q}_{h w, a}+\epsilon_{a} \sigma T_{w, a}{ }^{4}-\epsilon_{n} \sigma T_{w, n}{ }^{4}+ \\
& {\left[k_{c}(\partial T / \partial Y)\right]_{w, a}-\left[k_{c}(\partial T / \partial Y)\right]_{w, n} }
\end{aligned}
$$

where the subscripts $n$ and $a$ denote tests performed in nitrogen and air streams, respectively.

Comparable terms evaluated in air and nitrogen at a given wall temperature will now be considered. If the surface emissivity is assumed to be equal in both test gases, the reradiated energy terms are equivalent. The conduction terms $\left[k_{c}(\partial T / \partial Y)\right]_{w}$ are assumed to be equal on the basis of the following argument. Since energy conducted into the material is used in the decomposition process and in heating the product gases, one concludes that the vapor injection rate is a function of the heat conducted into the material. For the one-dimensional, steady-state, heat-conduction process this function can be represented by the equation

$$
\dot{m}_{v} \approx f\left[k_{c}(\partial T / \partial Y)\right]_{w} \approx f\left[\left(k_{c} / \delta_{c}\right)\left(T_{w}-T_{p}\right)\right]
$$

Now if the quantities $k_{c} / \delta_{c}$ and $T_{p}$ are independent of gas composition, then the mass injection rate of vapor will, in turn, be independent of gas composition and solely a function of wall temperature. This is shown to be the case by the correlation presented in Fig. 12. The difference in the energy balance between test gases of air and nitrogen at a

$\S$ Measurements of the external response of the material (i.e. the surface temperature was shown to reach an asymptotic value and the mass loss was found to vary linearly with time) indicate that a quasi-steady-state ablation had been established.

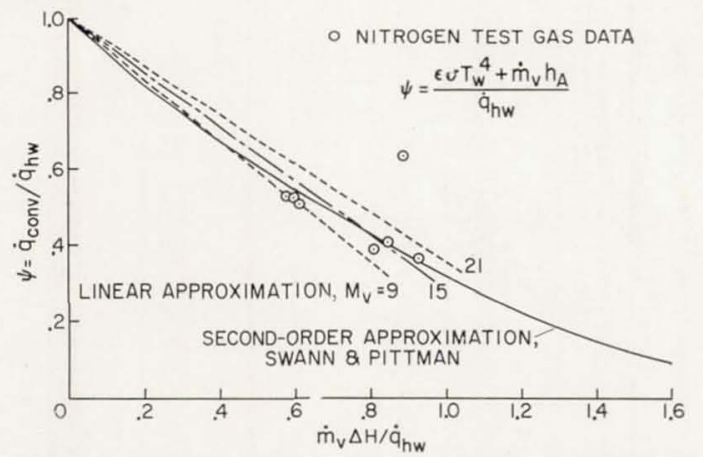

Fig. 11 Ratio of heat transfer with and without mass injection as a function of dimensionless injection rate.

given surface temperature thus reduces to

$$
\left.\dot{q}_{\mathrm{comb}, t}\right|_{T_{w}}=\left.\left(\psi_{n} \dot{q}_{h w, n}-\psi_{a} \dot{q}_{h w, a}\right)\right|_{T_{w}}
$$

The ratio of the total combustion heating to the net convective heating is obtained by dividing Eq. (A6) by $\psi_{a} \dot{q}_{h w, a}$ :

$$
\frac{\dot{q}_{\mathrm{comb}, t}}{\psi_{a} \dot{q}_{h w, a}}=\frac{\left(\psi_{n} / \psi_{a}\right) \dot{q}_{h w, n}-\dot{q}_{h w, a}}{\dot{q}_{h w, a}}
$$

The foregoing equation shows that the evaluation of the combustion heating rate requires a knowledge of the hot-wall heating rates and of $\psi_{n}$ and $\psi_{a}$. The hot-wall heating rates were deduced from measurements of cold-wall heating rate, wall temperatures, and stream enthalpy. The determination of the parameter $\psi$, described below, was based on considerations of linear and second-order correlatiens and on values deduced from the present data obtained in nitrogen.

A linear correlation for $\psi$ has resulted from a number of experimental and theoretical stagnation region studies ${ }^{21-23}$ and is given by

$$
\psi=1-\left(\beta \dot{m}_{v} \Delta H / \dot{q}_{h v o}\right)
$$

where $\dot{m}_{v}$ is the mass flux of the vapors from the surface, $\Delta H$ is the enthalpy potential across a nontranspiration-cooled boundary layer, and $\beta$ is the transpiration factor. The transpiration factor was also determined in these same studies and has the general form

$$
\beta=E\left(M_{\infty} / M_{v}\right)^{\alpha}
$$

where $M_{\infty}$ and $M_{v}$ are the molecular weights of the freestream gas and injected vapors, respectively, and $E$ and $\alpha$ are empirical constants. Values of $E$ and $\alpha$ are reported as $0.60 \leq$ $E \leq 0.72$ and $0.25 \leq \alpha \leq 0.4 .^{21-23}$

Determining the molecular weight of the injected vapors is the first problem one is faced with when analyzing transpiration cooling effects. For a relatively simple subliming material, such as teflon, the possible vapor products of decomposition are few, and it is generally estimated that the product is the $\mathrm{C}_{2} \mathrm{~F}_{4}$ monomer. ${ }^{21},{ }^{23}$ The problem is not

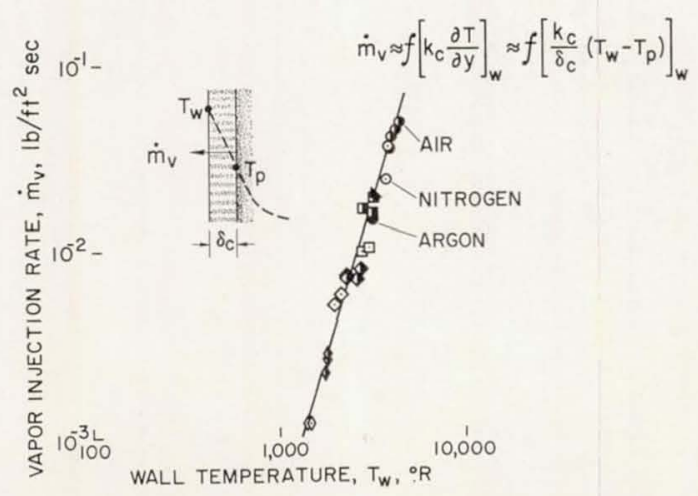

Fig. 12 Variation of vapor injection rate with wall temperature. 
well defined when one is considering a highly complex, crosslinked plastic material such as the phenolic nylon composite used in this investigation. The molecular weight of the injectants was estimated by first using an approximate chemical composition of the phenolic nylon material, second, evaluating the structure of the char as being carbonaceous with the density as heretofore determined, and third, examining the possible combinations of the resulting carbonhydrogen-oxygen-nitrogen mixture. It was found that the molecular weight could feasibly range from 9 to 21 , depending upon the amounts of each gaseous product formed $\left(\mathrm{N}_{2}\right.$, $\mathrm{H}_{2}, \mathrm{H}_{2} \mathrm{O}, \mathrm{CO}, \mathrm{C}_{2} \mathrm{H}_{2}, \mathrm{CH}_{4}$, and $\mathrm{NH}_{3}$ ). These calculations are in substantial agreement with the analytical predictions of the injectant molecular weight for a $50 \%$ nylon $50 \%$ phenolic composition presented in Ref. 24. The parameter $\beta$ was calculated from Eq. (A9) for molecular weights of 9, 15, and 21 , and $E=0.6$ and $\alpha=0.26$. The variations of $\psi$ with the dimensionless release rate $\dot{m}_{v} \Delta H / \dot{q}_{h w}$ for each value of $M_{v}$ was then determined from Eq. (A8) and are indicated in Fig. 11 as dashed lines.

The data points shown in the same figure were determined from the measurements obtained in nitrogen by using the energy balance, Eq. (A3), which was written in the form

$$
\psi=\frac{\epsilon \sigma T_{w}{ }^{4}+\left[k_{c}(\partial T / \partial Y)\right]_{w}}{\dot{q}_{h w}}
$$

where the heat-conduction term was related to the mass injection of vapors in the manner suggested by the correlation of Fig. 12 :

$$
\left[k_{c}(\partial T / \partial Y)\right]_{w}=\dot{m}_{v} h_{A}
$$

The quantity $h_{A}$, which is the amount of heat required to produce $1 \mathrm{lb}$ of vapor, was assumed to be $1000 \mathrm{Btu} / \mathrm{lb}$. To account for the loss of material from the sides of the model, tests were conducted with cored models. It was found that the stagnation-region injection rate was approximately 0.7 of the values shown in Fig. 6c.

A second-order approximation for $\psi$, developed by Swann and Pittman ${ }^{25}$ for charring ablators, was then calculated for a vapor molecular weight of 15 and is indicated by the heavy solid line in Fig. 11. This particular molecular weight was chosen because it resulted in the best agreement between the theory and the experiment. This correlation was used for both air and nitrogen in determining the combustion heating rate from Eq. (A7).

The foregoing analysis, in particular Eq. (A5), may be applied to the experimental results only if the heat-conduction process within the test models is, in fact, one-dimensional. One might question the validity of such an assumption when the data are obtained with small-scale models where twodimensional heat transfer might arise as a result of nonuniform heating of the test model, especially at the sides. However, in view of the following observations, it is concluded that such effects, if they exist, are small and thus do not seriously influence the ablation behavior of the test models at the stagnation point. First, the cold-wall heating distribution over the blunted test configuration has been shown to be relatively flat out to the corner region. Second, the effect of corner rounding on stagnation-region heating was investigated in separate tests of heat-transfer models having various corner radii and was found to be second order. Third, a post-run inspection of the ablation models, which had been encapsulated in epoxy and thin-sectioned, showed that the char thickness and decomposition along the sides of the models were minimal when compared to the stagnation-region values. Furthermore, the char formed at the sides, in contrast to the stagnation-region char, was found to be electrically nonconducting, and thus the char thermal conductivity is likely to be lower on the afterbody. Fourth the char was uniform in thickness along most of the face, which implies that heating conditions were constant over the face.

\section{References}

Lees, L., "Convective heat transfer with mass addition and chemical reactions," Third AGARD Combustion and Propulsion Colloquium, Palermo (Pergamon Press, New York, 1958), pp. 451-498.

${ }^{2}$ Scala, S. M., "The ablation of graphite in dissociated air, Part I: Theory,"' IAS Paper 62-154 (1962).

${ }^{3}$ Moore, J. A. and Zlotnik, M., "Combustion of carbon in an air stream," ARS J. 31, 1388-1397 (1961).

${ }^{4}$ Cohen, C. B., Bromberg, R., and Lipkis, R. P., "Boundary layers with chemical reactions due to mass addition," Jet Propulsion 28, 659-668 (1958).

${ }^{5}$ Hartnett, J. P. and Eckert, E. R. G., "Mass transfer cooling with combustion in a laminar boundary layer," Proceedings of the 1958 Heat Transfer and Fluid Mechanics Institute (Stanford University Press, Stanford, Calif., 1958), pp. 54-68.

${ }^{6}$ Scala, S. M. and Gilbert, L. M., "Thermal degradation of a char-forming plastic during hypersonic flight," ARS J. 32, 917-924 (1962).

${ }^{7}$ Chamberlain, D. L., Jr., Van Sickle, D. E., and Marynowski, C. W., "A study of the mechanism of ablation of reinforced plastics," Wright Air Dev. Center TR 59-668, Part II (September 1960).

${ }^{8}$ Sager, S. L. and Buhler, R. D., "Plasmajet wind tunnel ablation tests of Fiberglass Micarta 259-2 G. R. L," TR-19, Contract DA-04-495-ORD-1032, Army Ballistic Missile Agency (March 1959).

${ }^{9}$ Vojvodich, N. S. and Winkler, E. L., "The influence of heating rate and test stream oxygen content on the insulation efficiency of charring materials," NASA TN D-1889 (1963).

${ }^{10}$ Vojvodich, N. S., "The performance of ablative materials in a high energy, partially dissociated, frozen nitrogen stream," NASA TN D-1205 (1962).

${ }^{11}$ Winkler, E. L. and Griffin, R. N., Jr., "Effects of surface recombination on heat transfer to bodies in a high enthalpy stream of partially dissociated nitrogen," NASA TN D-1146 (1961).

${ }^{12}$ Winkler, E. L. and Griffin, R. N., Jr., "Measurements in a frozen, partially dissociated, high-speed gas stream," Proceedings of the Second Symposium on Hypervelocity Techniques (Plenum Press, New York, 1962), pp. 511-522.

${ }^{13}$ Fay, J. A. and Riddell, F. R., "Theory of stagnation point heat transfer in dissociated air," J. Aerospace Sci. 25, 73-85, 121 (1958).

${ }^{14}$ Inouye, M. and Lomax, H., "Comparison of experimental and numerical results for the flow of a perfect gas about bluntnosed bodies," NASA TN D-1426 (1962).

${ }^{15}$ Steg, L. and Lew, H., "Hypersonic ablation," Space Sciences Lab., General Electric Co., R62SD55, Class II (May 1962).

${ }^{16}$ Moore, D. G., "Investigation of shallow reference cavities for high-temperature emittance measurements," Symposium on Measurements of Thermal Radiation Properties of Solids (September 1962).

${ }^{17}$ Boison, J. C. and Curtiss, H. A., "An experimental investigation of blunt body stagnation point velocity gradient," ARS J. $29,130-135$ (1959).

${ }_{18}$ Scala, S. M. and Gilbert, L. M., "Aerothermochemical behavior of graphite at elevated temperatures," Graphite Symposium of the American Ceramic Society (October 1963).

${ }^{19}$ Zinman, W. G., "A study of the interaction between carbon and dissociated gases," Rept. R59SD452, General Electric Co., Missile and Space Vehicle Dept. (November 1959).

${ }^{20}$ Barber, E. A., "An experimental investigation of stagnation point injection," AIAA Preprint 63-433 (August 1963).

${ }^{21}$ Schmidt, D. L., "Thermal parameters of re-entry ablative plastics," Wright Air Dev. Div. TR 60-101 (1960).

${ }^{22}$ Georgiev, S., "The relative merits of various test facilities with regard to simulation of hypersonic ablation phenomena," Hypervelocity Techniques Symposium (October 1960).

${ }^{23}$ Adams, M. C., "Recent advances in ablation," ARS J. 29, 625-632 (1959).

${ }^{24}$ Kratsch, K. M., Hearne, L. F., and McChesney, H. R., "Thermal performance of heat shield composites during planetary entry," AIAA-NASA Meeting on Problems of Manned Interplanetary Exploration (September 1963).

${ }^{25}$ Swann, R. T. and Pittman, C. M., "Numerical analysis of the transient response of advanced thermal protection systems for atmospheric entry," NASA TN D-1370 (1962). 